\title{
Meeting polypoidal choroidal vasculopathy treatment needs halfway
}

Penny Lott Pooi Wah ${ }^{1,3}$, Rubamalar Gunatheesan ${ }^{1,4}$, Beau Fenner ${ }^{1,2}$, Gemmy Cheung Chui Ming ${ }^{1,2}$

${ }^{1}$ Singapore Eye Research Institute, Singapore National Eye Centre, Singapore; ${ }^{2}$ Duke-NUS Graduate Medical School, National University of Singapore, Singapore; ${ }^{3}$ Department of Ophthalmology, University of Malaya Eye Research Centre, Faculty of Medicine, University of Malaya, Kuala Lumpur, Malaysia; ${ }^{4}$ Tun Hussein Onn National Eye Hospital, Petaling Jaya, Selangor, Malaysia

Polypoidal choroidal vasculopathy (PCV) is a subtype of neovascular age-related macular degeneration (nAMD) that accounts for up to $50 \%$ of nAMD cases in Asia. ${ }^{1}$ The PCV complex comprises polypoidal lesions $(\mathrm{PL})$ and branching neovascular network (BNN), both of which are found between the retinal pigment epithelium (RPE) and the outer portion of Bruch's membrane. ${ }^{2}$ Hence, $\mathrm{PCV}$ should be considered a variant of type 1 neovascularization. ${ }^{3}$ In addition, studies of the choroidal background suggest PCV resides within the pachychoroid spectrum of diseases, characterized by choroidal thickening, dilated Haller's layer vessels, and attenuation of the overlying choriocapillaris. ${ }^{4}$

Vision loss in PCV may occur via several mechanisms, including exudation and haemorrhage. ${ }^{2,3}$ While anti-VEGF monotherapy has been demonstrated to reduce retinal thickness, closure of PLs is often desired as unclosed PLs are often the source of hemorrhage..$^{2,5,6}$ Photodynamic therapy (PDT) has been shown to be superior to ranibizumab monotherapy in resulting in closure of PLS in the EVEREST and EVEREST II studies. ${ }^{5}$ On the other hand, anti-VEGF monotherapy has been shown to be effective in preserving vision in PCV patients, but its ability to close PLs appears variable. ${ }^{7}$

In the EVEREST II study, combination therapy with PDT was superior to ranibizumab monotherapy in achieving closure of PLs. ${ }^{55}$ The combination arm also required less retreatment. However, in clinical practice, some concerns remain regarding the potential adverse effects of full-fluence PDT, which may include choroidal ischemia and RPE disruption. Severe vision loss following full-fluence PDT has been reported in approximately $1 \%$ of cases. ${ }^{8}$ In light of this risk, modified PDT, either as half-fluence PDT (hfPDT; light dose of $25 \mathrm{~J} / \mathrm{cm}^{2}$ ) or half-dose PDT 
(hdPDT; $3 \mathrm{mg} / \mathrm{m}^{2}$ verteporfin) have been attempted in the management of PCV. ${ }^{6}$ In this issue, Chow and coworkers reported outcomes for hdPDT combination therapy and anti-VEGF monotherapy for PCV. In their retrospective case series, 6-month functional and structural outcomes were reported for eight eyes treated with hdPDT combined with ranibizumab or aflibercept, and for 10 eyes treated with ranibizumab or aflibercept monotherapy. The authors reported a trend towards better letter gains in the combination therapy arm, with similar anti-VEGF treatment burden in both groups. PL closure rate and choroidal thickness changes post-treatment were not reported. Perhaps counterintuitively, reduction in central subfield thickness was more marked in the monotherapy group, although not statistically significant. These somewhat conflicting findings may reflect the heterogeneity in lesion characteristics. As the authors have rightly pointed out, the combination group may have preselected patients with more aggressive PCV, or cases with more extensive disease or aggressive morphology. In the hdPDT group, there was also a mixture of deferred or prompt combination. This variation in timing of hdPDT may also affect the findings.

In addition to reporting vision outcome and treatment number, an additional endpoint which would be important to note in this study is the polyps' status. The speed of complete PLs closure rate can be evaluated as one of the biomarkers of treatment outcome. Patients with complete PL closure might require less anti-VEGF and thus reduce the treatment burden. Choroidal anatomy plays an important role in PCV pathophysiology, changes in choroidal thickness following hdPDT. Poorer anatomical response to loading dose in anti-VEGF in a thicker choroid have been reported. ${ }^{9}$

The current work by Chow and colleagues suggests the efficacy of hdPDT in PCV remains unclear, although a favourable safety profile was seen in their series. These results highlight the difficulty in generalizing treatment recommendations as PCV lesions are highly heterogenous. hdPDT combined with ranibizumab was previously reported to be effective for smaller PCV lesions but less effective in cases with BNN and multiple PLs. ${ }^{10}$

In real-world settings, complete regression of PLs is a desirable endpoint for patients with PCV as it decreases the frequency of retreatment and reduces the risk of devastating haemorrhage and chronic exudation. Several methods have been shown to decrease the rate of PLs, including prompt combination at baseline with either full-fluence or hfPDT, prolonged monthly loading with anti-VEGF for up to 6 months, or deferred combination with PDT. Future studies evaluating the efficacy and safety of these treatment modalities will need to carefully balance the baseline characteristics of PCV lesions and choroidal background. Longer follow-up design will also be necessary to evaluate any differential effect on recurrence rate. Until then, for clinical purposes, reviewing the indocyanine green angiography features at month 3 remains an important assessment, especially in persistent, recalcitrant, aggressive disease that progress despite initial monthly anti-VEGF therapy. 


\section{References}

1. Wong CW, Yanagi Y, Lee WK, et al. Age-related macular degeneration and polypoidal choroidal vasculopathy in Asians. Prog Retin Eye Res. 2016;53:107-39.

2. Cheung CMG, Lai TYY, Ruamviboonsuk P, et al. Polypoidal Choroidal Vasculopathy: Definition, Pathogenesis, Diagnosis, and Management. Ophthalmology. 2018.

3. Cheung CMG, Lee WK, Koizumi H, et al. Pachychoroid disease. Eye (Lond) .2019;33(1):14-33.

4. Lee WK, Baek J, Dansingani KK, et al. Choroidal Morphology in Eyes with Polypoidal Choroidal Vasculopathy and Normal or Subnormal Subfoveal Choroidal Thickness. Retina. 2016;36 Suppl 1:S73-S82.

5. Koh A, Lee WK, Chen LJ, et al. EVEREST study: efficacy and safety of verteporfin photodynamic therapy in combination with ranibizumab or alone versus ranibizumab monotherapy in patients with symptomatic macular polypoidal choroidal vasculopathy. Retina. 2012;32(8):1453-64.

6. Ngo WK, Chee WK, Tan CS, Lim TH. Comparing efficacy of reduced-fluence and standard-fluence photodynamic therapy in the treatment of polypoidal choroidal vasculopathy. BMC Ophthalmol. 2020;20(1):150.

7. Fenner BJ, Cheung CMG, Sim SS, et al. Evolving treatment paradigms for PCV. Eye (Lond). 2021.

8. Wormald R, Evans J, Smeeth L, Henshaw K. Photodynamic therapy for neovascular age-related macular degeneration. Cochrane Database Syst Rev. 2007(3):Cd002030.

9. Kim H, Lee SC, Kwon KY, Lee JH, Koh HJ, Byeon SH, et al. Subfoveal thickness as a predictor of treatment response to anti-vascular endothelial growth factor therapy for polypoidal choroidal vasculopathy. Graefes Arch Clin Exp Ophthalmol. 2016;254:1497-503.

10. Wong IY, Shi X, Gangwani R, et al. 1-year results of combined half-dose photodynamic therapy and ranibizumab for polypoidal choroidal vasculopathy. BMC Ophthalmol. 2015;15:66. 